\title{
SORÇÃO/DESSORÇÃO E MINERALIZAÇÃO DO INSETICIDA ACEFATO EM SOLO
}

MARA MARCHETTI * LUIZ CARLOS LUCHINI **

\begin{abstract}
Estudou-se a adsorção/dessorção e mineralização do inseticida organofosforado acefato aplicado em solo tropical (argissolo) da região de Campinas-SP (Brasil). Foram usadas técnicas radiométricas nas análises do solo, do ar e da solução aquosa quanto à presença de ${ }^{14} \mathrm{C}$-acefato no solo tratado. Isotermas de adsorção e dessorção foram obtidas tratando-se 1,2 e $4 \mathrm{~g}$ de solo com $10 \mathrm{~mL}$ de solução aquosa de $\mathrm{CaCl}_{2}$ 0,01 mol.L-1 com 7 diferentes concentrações de acefato. Após $24 \mathrm{~h}$ de agitação para atingir o equilíbrio solo/solução, a adsorção foi determinada nos extratos obtidos mediante leitura da atividade do ${ }^{14} \mathrm{C}$-acefato por contagem de cintilação em líquido. Os parâmetros, constante de adsorção/dessorção (K) e constante exponencial $(\boldsymbol{n})$ da equação de Freundlich foram obtidos mediante regressão linear, além da constante de distribuição $\left(\mathbf{K}_{\mathrm{d}}\right)$ e do coeficiente de adsorção normalizado para conteúdo de carbono orgânico $\left(\mathbf{K}_{\mathrm{oc}}\right)$. O estudo da mineralização do acefato no solo foi conduzido em frascos biométricos de Bartha, com $50 \mathrm{~g}$ de solo e $10 \mathrm{~mL}$ de $\mathrm{KOH}$ 0,2 mol.L-1 como solução de captura de ${ }^{14} \mathrm{CO}_{2}$. Acompanhou-se a cinética da degradação por meio de dois experimentos com duração de 14 e 64 dias. Alíquotas da solução-armadilha e subamostras do solo, após combustão, foram analisadas por contagem de cintilação em líquido. Os resultados das isotermas de adsorção obtidas para o acefato revelaram que 0 composto é pouco retido em argissolo (as constantes $\boldsymbol{K}_{f}$ e $\mathbf{K}_{\mathrm{d}}$ apresentaram valores menores que 5 ; $\mathbf{K}_{\text {oc }}$ foi de 56). Os valores dos coeficientes de dessorção indicam processo lento e não-completo durante o período do experimento. O resultado da mineralização indicou que o acefato é facilmente degradado nos 10 primeiros dias, com cerca de $70 \%$ do inseticida desprendido na forma de ${ }^{14} \mathrm{CO}_{2}$. Sua meia-vida no solo apresentou os valores de 18,9 e 1.386 dias.
\end{abstract}

PALAVRAS-CHAVE: SOLO-DEGRADAÇÃO; INSETICIDAS; TÉCNICAS RADIOMÉTRICAS.

* $\quad$ Mestre em Ciências, Instituto Biológico (IB), São Paulo, SP (e-mail: mmarchet@bol.com.br).

** Pesquisador Científico do Instituto Biológico, Laboratório de Ecologia de Agroquímicos, Centro de Proteção Ambiental, São Paulo, SP, Brasil (email: luchini@biologico.sp.gov.br). 


\section{INTRODUÇÃO}

O destino dos agrotóxicos é determinado pela retenção, transporte e transformação desses compostos químicos liberados no meio ambiente. A retenção, o transporte e, indiretamente, a transformação são processos basicamente influenciados pelo fenômeno denominado de sorção. A sorção pode influenciar de forma bastante significativa a lixiviação, importante processo de transporte, no qual os agrotóxicos alcançam os recursos hídricos subterrâneos afetando sua qualidade.

O fenômeno de sorção decorre da partição do agrotóxico entre as fases sólida e a solução do solo, sendo dependente das propriedades fisico-químicas dos colóides do solo e do agrotóxico. Pode ser classificado como sorção química, na qual estão envolvidas energias de ativação do processo com transformação dos compostos, e/ou sorção física, cujas energias são mais baixas e a natureza química das espécies envolvidas é preservada (BURCHILL, HAYES e GREENLAND, 1981; LAVORENTI, 1996).

Normalmente, a sorção no sistema solo-agrotóxico é do tipo físico (HAQUE, 1975). Freqüentemente, o termo sorção é substituído por adsorção para definir o fenômeno de retenção. $O$ fenômeno inverso é chamado de dessorção, responsável por liberar o agrotóxico dos colóides do solo e permitir sua transformação ou transporte. A mudança na estrutura molecular de compostos orgânicos (SOMASUNDARAN e COATS, 1991; LAVORENTI, 1996) pode ocorrer mediante processos biológicos ou não-biológicos. As reações de transformação envolvendo microrganismos são normalmente os únicos processos pelos quais o composto pode ser mineralizado, isto é, convertido a $\mathrm{CO}_{2}, \mathrm{H}_{2} \mathrm{O}$ e outros íons (SCHWARZENBACH, GSCHWEND e IMBODEM, 1993b; SCHEUNERT, 1993).

$O$ agrotóxico escolhido para o estudo foi o acefato, inseticida organofosforado encontrado comercialmente no Brasil com os nomes de Acefato Fersol, Cefanol, Evolution e Orthene. Seu principal metabólito (Figura 1), o metamidofós, também apresenta propriedades inseticidas (MARCHETTI, 2001).

\section{FIGURA 1 - FÓRMULAS ESTRUTURAIS DO ACEFATO E SEU METABÓLITO}<smiles>COP(=O)(NC(C)=O)SC</smiles>

Acefato<smiles>COP(N)(=O)SC</smiles>

Metamidofós

O acefato apresenta alta solubilidade em água e sofre hidrólise em meio alcalino (SCARONI e CALDARI, 1997). Seu coeficiente de partição octanol-água e o de partição matéria orgânica-água indicam que o composto é pouco adsorvido aos colóides do solo, evidenciando alto potencial de lixiviação. Contudo, a meia-vida do composto relatada em literatura é de poucos dias, aumentando com o abaixamento do $\mathrm{pH}$ do solo (MONTGOMERY, 1993).

Procurou-se neste trabalho avaliar, mediante técnicas radiométricas, as interações do acefato com solo tropical sob o ponto de vista de sua adsorção e degradação.

\section{MATERIAL E MÉTODOS}

\subsection{SOLO}

Coletou-se o solo utilizado neste experimento na estação experimental do Instituto Biológico em Campinas. As amostras coletadas na superfície $(0$ a $20 \mathrm{~cm})$ foram passadas em peneira de malha 
2,0 mm e utilizadas para os testes conduzidos em laboratório. Determinou-se, previamente, a umidade do solo em balança para determinação de peso seco Mettler LJ16. As características fisico-químicas do solo foram determinadas pelo Departamento de Solos e Nutrição de Plantas da Escola Superior de Agricultura "Luiz de Queiroz" (ESALQ/USP, Piracicaba).

O solo, classificado como argissolo vermelho-amarelo que sofreu modificações antrópicas, apresentou $\mathrm{pHCaCl}$ ácido $(5,2)$ e baixo teor de matéria orgânica $\left(19 \mathrm{~g} \cdot \mathrm{dm}^{-3}\right)$. É pobre em nutrientes, com fração silte + argila pouco maior que a fração arenosa (teores de $470 \mathrm{~g} \cdot \mathrm{Kg}^{-1}$ areia, $190 \mathrm{~g} \cdot \mathrm{Kg}^{-1} \mathrm{de}$ silte e $340 \mathrm{~g} . \mathrm{Kg}^{-1}$ de argila), sendo classificado como de textura média.

\subsection{AGROTÓXICO}

O acefato marcado com carbono-14, obtido da "International Isotopes Münich" (Alemanha), apresentava atividade de 55,5 MBq e atividade específica de 625,19 MBq. mmol ${ }^{-1}\left(16,9 \mathrm{mCi} \mathrm{mmol}^{-1}\right) . \mathrm{O}$ produto foi purificado no Laboratório de Ecologia de Agroquímicos (Instituto Biológico) antes de ser utilizado nos experimentos.

Acefato grau técnico com pureza de 97\%, fornecido pela "Sipcam Agro" (Itália), foi utilizado como substância carreadora.

\subsection{TESTE DE ADSORÇÃO/DESSORÇÃO}

O ensaio de adsorção/dessorção foi realizado com 1, 2 e $4 \mathrm{~g}$ de solo, soluções com diferentes concentrações do acefato grau técnico e seu correspondente radiomarcado $(0 ; 0,2 ; 0,5 ; 1 ; 2 ; 4 ; 10$ e $\left.20 \mu \mathrm{g} \cdot \mathrm{mL}^{-1}\right)$, tendo solução aquosa de $\mathrm{CaCl}_{2} 0,01 \mathrm{~mol}^{\mathrm{L}} \mathrm{L}^{-1}$ como eletrólito-suporte. Após agitação do sistema solo/solução por 24 horas, $1 \mathrm{~mL}$ do sobrenadante de cada tubo foi analisado (em triplicata) por contagem de cintilação em líquido (CCL) para determinar $0{ }^{14} \mathrm{C}$-acefato adsorvido ou dessorvido.

As isotermas de adsorção e dessorção foram obtidas utilizando-se o modelo matemático de Freundlich: $\boldsymbol{A}=\boldsymbol{K}_{f} \boldsymbol{C} \boldsymbol{e}^{n}$, em que $\boldsymbol{A}$ é a quantidade de inseticida adsorvido ou remanescente após dessorção ( $\mu \mathrm{g} \cdot \mathrm{g}^{-1}$ de solo); $C e$ é a concentração do inseticida na solução após o equilíbrio ( $\left.\mu \mathrm{g} \cdot \mathrm{mL}^{-1}\right) ; \boldsymbol{K}_{f}$ (coeficiente de adsorção/ dessorção de Freundlich) e $\boldsymbol{n}$ são parâmetros de regressão característicos para cada sistema solo-substância estudado (RAO e JESSUP, 1983; LAVORENTI, 1996; LUCHINI, 1987 e 1997).

O parâmetro $K_{f}$ foi analisado de acordo com a tabela descrita nas normas para avaliação de agentes químicos (BRASIL, 1993). O parâmetro $K_{d}$ (constante de distribuição em dada concentração), próprio para a comparação da capacidade adsortiva entre solos (HANCE, 1988) foi calculado assumindose $\boldsymbol{n}=1$ para a concentração no equilíbrio $(C e)$ de $20 \mu \mathrm{g} \cdot \mathrm{mL}^{-1}$. Outro coeficiente calculado foi $\mathrm{K}_{\mathrm{oc}}$ (coeficiente de adsorção normalizado) em relação ao conteúdo de carbono orgânico do solo. Essa constante é segundo RAO e JESSUP (1983) mais adequada para comparação da adsorção de determinado agrotóxico em diferentes tipos de solo.

\subsection{TESTE DE MINERALIZAÇÃO}

O solo para o estudo de mineralização foi reativado pela adição de água até $40 \%$ de sua capacidade máxima de retenção de água. A seguir, foi mantido a $25^{\circ} \mathrm{C}$ por 1 semana em estufa incubadora no escuro.

Subamostras de $50 \mathrm{~g}$, em peso seco, de solo reativado e homogeneizado após aplicação de solução com acefato radiomarcado e acefato técnico foram colocados em frascos biométricos (BARTHA 
e PRAMER, 1965). Esses continham $10 \mathrm{~mL}$ de solução de $\mathrm{KOH} 0,2 \mathrm{~mol} \cdot \mathrm{L}^{-1}$ para a captura do ${ }^{14} \mathrm{CO}_{2}$ desprendido na mineralização do ${ }^{14} \mathrm{C}$-acefato .

O teste foi realizado pelo período de 64 dias, sendo as coletas da solução-armadilha de $\mathrm{CO}_{2}$ inicialmente diárias, depois a cada 3 dias e finalmente semanais. Alíquotas de $1 \mathrm{~mL}$ em triplicata da solução-armadilha foram analisadas por contagem de cintilação em líquido (CCL).

Após $0,3,7,14,28$ e 64 dias, 6 subamostras de cerca $500 \mathrm{mg}$ de solo de cada frasco foram submetidas à combustão para quantificação de ${ }^{14} \mathrm{C}$-acefato ou ${ }^{14} \mathrm{C}$-metabólitos remanescentes. $\mathrm{O}$ restante do solo dos frascos biométricos foi submetido à extração com mistura de solventes (metanol:água, 95:5, v/v) por $16 \mathrm{~h}$. O sobrenadante foi retirado e alíquotas de $1 \mathrm{~mL}$, em triplicata, foram analisadas por CCL para quantificação dos resíduos extraíveis. O solo submetido à extração foi seco e subamostras de $500 \mathrm{mg}$, em triplicata, foram submetidas à combustão e análise por CCL para quantificação dos resíduos ligados.

O segundo teste foi realizado durante 14 dias, visando complementar o teste anterior devido ao rápido processo de mineralização e para melhor aproximação da curva de desprendimento de $\mathrm{CO}_{2}$. As coletas da solução-armadilha foram realizadas em intervalos de 2 horas na primeira semana e de 4 horas, na segunda. As análises por CCL foram efetuadas conforme descrito anteriormente.

Calculou-se a meia-vida $\left(\mathbf{T}_{1 / 2}\right)$ do inseticida pela equação:

$$
T_{1 / 2}=\frac{0,693}{\lambda}
$$

O coeficiente de degradação $\lambda$ foi obtido em função da porcentagem do ${ }^{14} \mathrm{C}$-inseticida remanescente no solo, mediante regressão não-linear do tipo bi-exponencial.

\section{RESULTADOS E DISCUSSÃO}

\subsection{TESTE DE ADSORÇÃO/ DESSORÇÃO}

As isotermas de adsorção do acefato foram obtidas graficamente, plotando-se a concentração do acefato em equilíbrio (Ce) versus a quantidade do acefato adsorvido (As) para cada relação solo: solução (Figura 2).

\section{FIGURA 2 - ISOTERMAS DE ADSORÇÃO PARA $1 \mathrm{~g}, 2 \mathrm{~g}$ E $4 \mathrm{~g}$ DE SOLO NAS VÁRIAS CONCENTRAÇÕES DE ACEFATO EM SOLUÇÃO (Ce)}
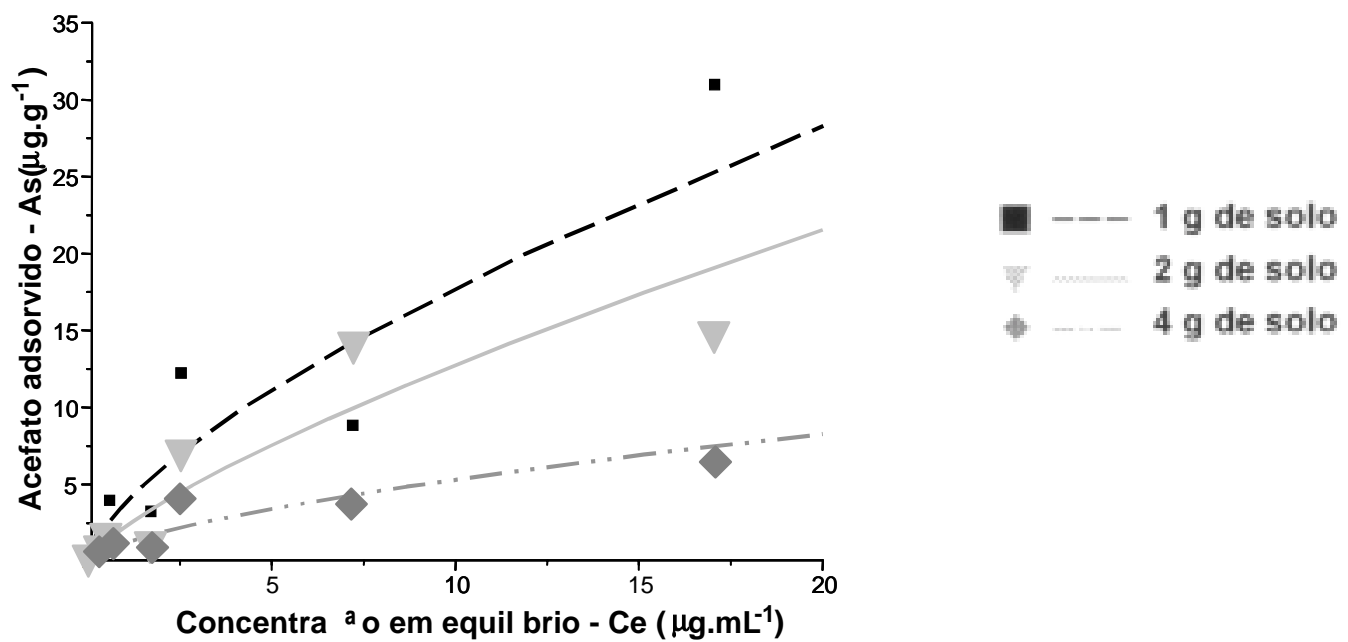
Observou-se que a quantidade de solo afetou a isoterma de adsorção. Quanto maior a quantidade de solo, menor a quantidade de acefato adsorvida. A relação é explicada pelo fato de alguns solos aumentarem a afinidade pelo composto quando se aumenta a concentração do mesmo (SCHWARZENBACH, GSCHWEND e IMBODEN, 1993a). As isotermas de adsorção do acefato para cada concentração solo-solução ajustaram-se à equação de Freundlich com $\mathrm{R}$ acima de 0,94 . Os coeficientes calculados $\boldsymbol{K}_{f}$ e $\boldsymbol{n}$ das isotermas de adsorção encontram-se na Tabela 1. O coeficiente de adsorção de Freundlich $\left(\boldsymbol{K}_{f}\right)$ próximo a 4 caracteriza pequena retenção do inseticida de acordo com a Secretaria Especial do Meio Amiente (BRASIL, 1993). A retenção de um agrotóxico no meio em que foi introduzido depende das propriedades fisico-químicas do meio e do agrotóxico. Quando baixa pode representar grande potencial de lixiviação do mesmo no perfil do solo. Esse dado deve ser confrontado com a solubilidade e persistência, entre outras características do agrotóxico.

Os valores obtidos para $\boldsymbol{K}_{f}$ estão de acordo com diversos autores e confirmam a baixa retenção do inseticida. RODRÍGUEZ-GONZALO, SÁCHEZ-MARTIN e SÁNCHEZ-CAMAZANO (1991) encontraram valores de cerca de 0,05 a 3,56, ARIENZO et al. (1993) de 0,5 a 3,0, enquanto que os valores observados por SÁNCHEZ-CAMAZANO et al. (1994) não ultrapassaram 0,9.

TABELA 1 - VALORES DO COEFICIENTE DE ADSORÇÃO/DESSORÇÃO $\left(K_{F}\right)$, O COEFICIENTE LINEAR DA ISOTERMA ( $M$ ) DE FREUNDLICH, DO COEFICIENTE DE DISTRIBUIÇÃO $\left(K_{\mathrm{D}}\right)$ E DO COEFICIENTE DE DISTRIBUIÇÃO NORMALIZADO $\left(\mathrm{K}_{\mathrm{oc}}\right)$

\begin{tabular}{|c|c|c|c|c|c|c|c|c|c|c|}
\hline & \multicolumn{3}{|c|}{$K_{f}$} & \multicolumn{3}{|c|}{$n$} & \multicolumn{3}{|c|}{$\mathbf{K}_{\mathrm{d}}$} & \multirow{2}{*}{$\frac{K_{o c}}{1 \mathrm{~g}}$} \\
\hline & $1 \mathrm{~g}$ & $2 \mathrm{~g}$ & $4 \mathrm{~g}$ & $1 \mathrm{~g}$ & $2 \mathrm{~g}$ & $4 \mathrm{~g}$ & $1 \mathrm{~g}$ & $2 \mathrm{~g}$ & $4 \mathrm{~g}$ & \\
\hline Adsor a o & 3,8 & 2,3 & 1,2 & 0,7 & 0,8 & 0,7 & 1,83 & 0,86 & 0,37 & 55,9 \\
\hline Dessor $\stackrel{a}{\circ} 01$ & 72,4 & 21,7 & 7,7 & 0,8 & 0,8 & 0,8 & & & & \\
\hline Dessor $\underline{a}$ o II & 804,5 & 284,6 & 27,4 & 0,9 & 1,0 & 0,8 & & & & \\
\hline Dessor $\underline{a}$ o III & 31,1 & 83,3 & 41,0 & 0,2 & 0,9 & 0,8 & & & & \\
\hline
\end{tabular}

RODRÍGUEZ-GONZALO et al. (1991) sugerem que os maiores valores de $\boldsymbol{K}_{f}$ para o acefato sejam influenciados pelo maior teor de matéria orgânica $(M O)$ do solo e pelo grau de humificação dessa. Outros autores (ARIENZO et al., 1993 e SÁNCHEZ-CAMAZANO et al., 1994) sugerem que os valores maiores de $K_{f}$ estejam relacionados com o maior teor de silte+argila e conteúdo de smectita, mineral com grande capacidade adsortiva. Como o solo estudado apresentou baixo teor de $\mathrm{MO}$, a fração silte+argila pode ter influenciado no $K_{f}$ encontrado.

IGLESIAS-JIMÉNEZ, SÁNCHEZ-MARTIN e SÁNCHEZ-CAMAZANO (1996) também encontraram $K_{f}$ baixo (de 0,72 , com $n=0,93$ ) para o acefato em cambissolo temperado básico e eutrófico com baixo teor de matéria orgânica $\left(7,7 \mathrm{~g} . \mathrm{Kg}^{-1}\right)$ e fração arenosa maior que a de silte+argila. Tal resultado confirma a não-correlação entre acefato e MO verificada por SÁNCHEZ-CAMAZANO et al. (1994).

$O$ coeficiente $\mathbf{K}_{d}$ obtido quando se observa uma só concentração do agrotóxico no equilíbrio (nesse caso, $\mathrm{C}_{\mathrm{e}}=20 \mu \mathrm{g} \cdot \mathrm{mL}^{-1}$ de acefato na solução), que reflete os valores da constante anterior, também revela a baixa retenção do inseticida no solo (Tabela 1). $O$ valor de $K_{d}$ para $4 \mathrm{~g}$ de solo está em conformidade com o encontrado por YEN, LIN e WANG (2000) com $5 \mathrm{~g}$ para dois solos chineses. Esses, apesar de conterem grande fração silte+argila $\left(80\right.$ e $\left.88,1 \mathrm{~g} \mathrm{Kg}^{-1}\right)$ e baixo teor de matéria orgânica (1,76 e 2,34\%) apresentaram coeficientes de distribuição em torno de 0,39.

Comparando a adsorção do acefato com a do diazinon, também da família dos organofosforados, verificou-se comportamento distinto. O diazinon, normalmente, é mais retido que os acefato nos mesmos solos (ARIENZO et al., 1993; IGLESIAS-JIMÉNEZ, SÁNCHEZ-MARTIN e SÁNCHEZ-CAMAZANO, 
1996). Isto pode ser explicado pelas características particulares de sua molécula, como menor solubilidade em água e peso molecular cerca de três vezes maior que o do acefato.

As isotermas de dessorção (Figura 3) foram obtidas plotando-se a nova concentração de acefato, encontrada após o processo de dessorção (Ce'), e a quantidade de acefato remanescente do processo de adsorção (As).

\section{FIGURA 3 - ISOTERMAS DE DESSORÇÃO PARA CADA RELAÇÃO SOLO:SOLUÇÃO}
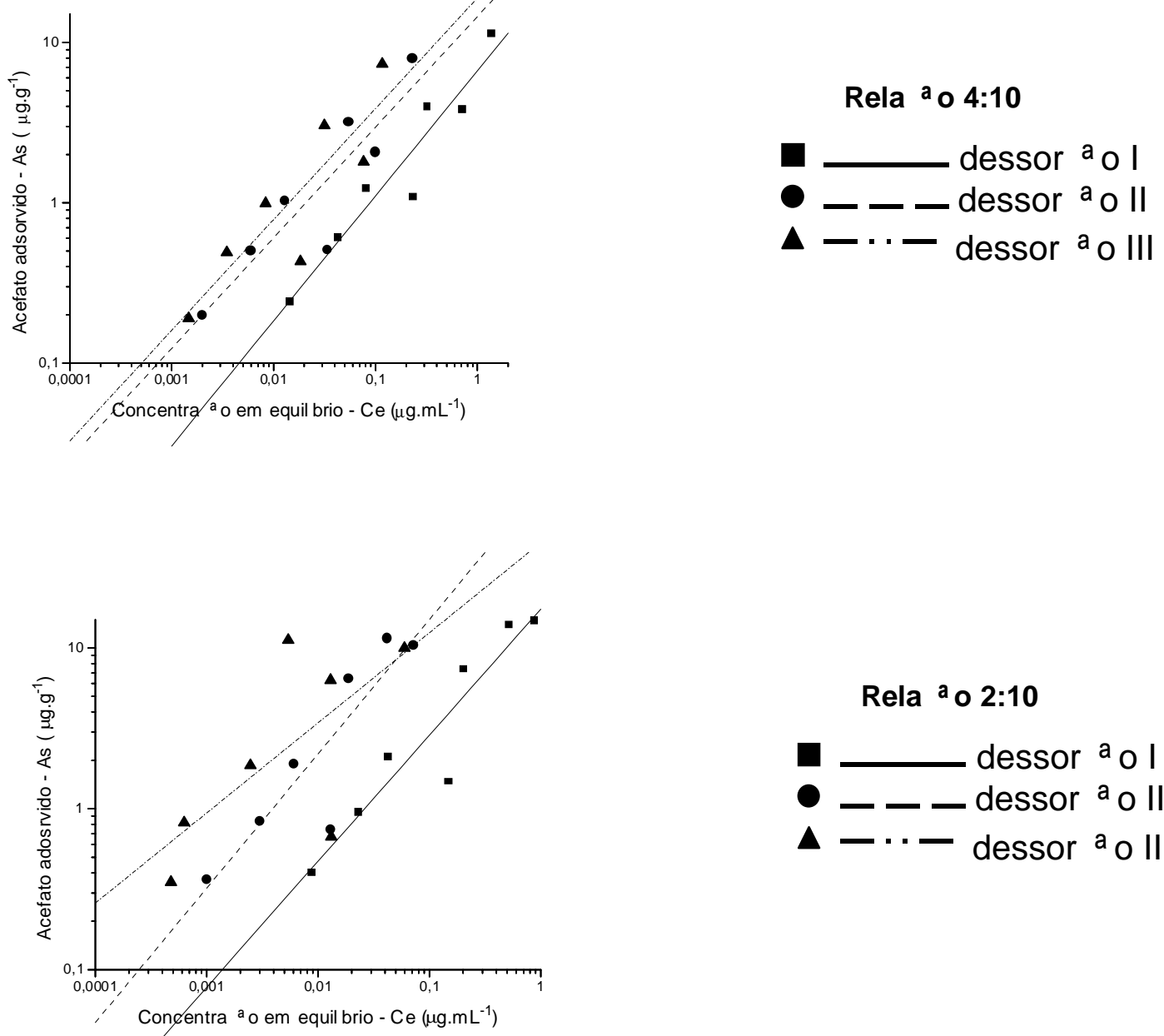

Rela a o 2:10

- dessor $\stackrel{\text { a }}{0}$ I

- - - - dessor $\stackrel{\text { a }}{0}$ II

$\Delta-\cdots-$ dessor $\stackrel{\text { a }}{\mathrm{A}}$ III

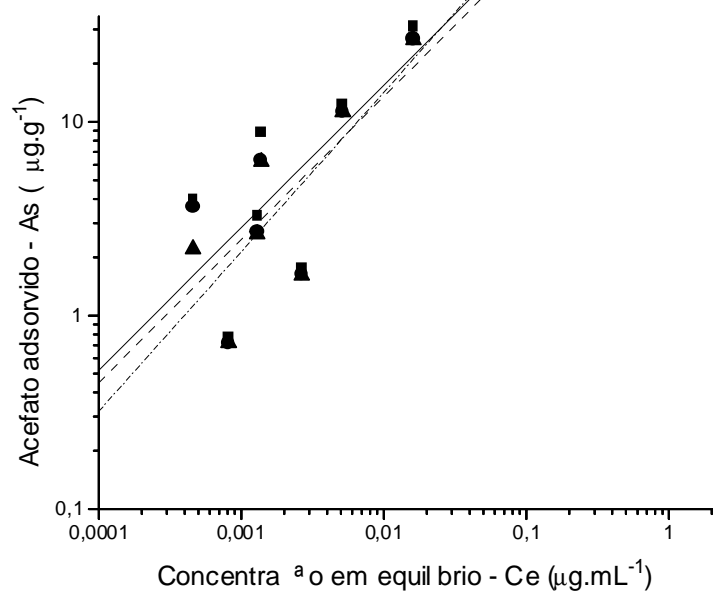

Rela $\stackrel{\text { a }}{\circ}$ 1:10

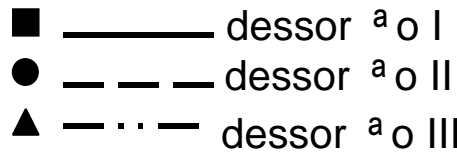


O ajuste das isotermas de dessorção à equação de Freundlich forneceu R acima de 0,90 e valores de coeficientes $\boldsymbol{K}_{\boldsymbol{f}}$ superiores, como pode ser visto na Tabela 1.

A dessorção de compostos químicos por água ou eletrólitos na maioria dos casos não é completa, ou seja, a adsorção não é completamente reversível. A parte não dessorvida se deve à fortes ligações que são quebradas somente pela extração com solventes, ou à ligações que levam a formação de "resíduos ligados" (SCHEUNERT, 1993). Segundo HANCE (1988), as isotermas de dessorção são mais difíceis de descrever. O fenômeno é normalmente mais lento que a adsorção, podendo provocar erros significativos causados pela decomposição do sorbato e modificação do sorbente devido ao período experimental.

Os valores obtidos para o coeficiente de adsorção $\left(\boldsymbol{K}_{\mathrm{f}}\right)$, observando-se o processo de dessorção, indicam que o inseticida estava fortemente ligado ao solo (alta retenção) mesmo após três ciclos de dessorção com solução aquosa de $\mathrm{CaCl}_{2}$. O processo de adsorção não foi completamente reversível, pois ainda permaneciam resíduos do inseticida no solo (Tabela 2). Esse fato foi também observado por LUCHINI, LORD e RÜEGG (1981) em estudo sobre a adsorção e dessorção de pesticidas em solos brasileiros. KOSKINEN e CHENG (1983) atribuíram a reversibilidade incompleta da adsorção à histerese. Essa, pode ser descrita por parâmetros termodinâmicos, tais como energia de ativação e calor de adsorção, que governam a dessorção e podem ser quantitativamente diferentes daqueles que afetam a adsorção. Há ainda, fatores como mudança no mecanismo de adsorção ou processos de degradação durante o experimento, conforme relatado por LUCHINI (1987 e 1997).

Os resíduos remanescentes no solo após as sucessivas dessorções foram quantificados por combustão e análise por CCL (Tabela 2).

\section{TABELA 2 - RESÍDUOS ENCONTRADOS NO SOLO APÓS AS DESSORÇÕES EM RELAÇÃO AO INICIALMENTE APLICADO}

\begin{tabular}{|c|c|c|c|}
\hline \multirow{2}{*}{$\begin{array}{c}\text { Concentra }{ }^{a} \text { o na } \\
\text { Solu a o }\end{array}$} & \multicolumn{3}{|c|}{ Res duos } \\
\hline & $1 \mathrm{~g}$ & $2 \mathrm{~g}$ & $4 \mathrm{~g}$ \\
\hline & \multicolumn{3}{|c|}{---- $(\% \pm$ desvio padra o) } \\
\hline 0 & 0,0 & 0,0 & 0,0 \\
\hline 0,2 & $9,55 \pm 1,03$ & $9,41 \pm 1,53$ & $10,10 \pm 0,91$ \\
\hline 0,5 & $8,26 \pm 0,46$ & $11,79 \pm 0,55$ & $8,14 \pm 0,51$ \\
\hline 1 & $9,76 \pm 1,13$ & $12,41 \pm 1,52$ & $9,56 \pm 0,58$ \\
\hline 2 & $5,11 \pm 0,97$ & $4,38 \pm 0,24$ & $4,93 \pm 1,04$ \\
\hline 4 & $7,83 \pm 1,07$ & $10,41 \pm 0,88$ & $8,37 \pm 0,41$ \\
\hline 10 & $2,67 \pm 0,32$ & $6,43 \pm 0,85$ & $3,24 \pm 0,33$ \\
\hline 20 & $1,34 \pm 0,14$ & $1,76 \pm 0,23$ & $1,70 \pm 0,19$ \\
\hline
\end{tabular}

\subsection{TESTE DE MINERALIZAÇÃO}

A Figura 4 evidencia a rápida degradação do acefato radiomarcado nos primeiros 10 dias e patamar com desprendimento lento de ${ }^{14} \mathrm{CO}_{2}$ até os 57 dias de incubação. A ação microbiana é, normalmente, a única que leva à mineralização completa de um xenobiótico (SCHEUNERT, 1993; SCHWARZENBACH, GSCHWEN e IMBODEN, 1993b). Verificou-se a produção de cerca $63 \%$ de $\mathrm{CO}_{2}$ ao longo do período de estudo (Figura 3). 


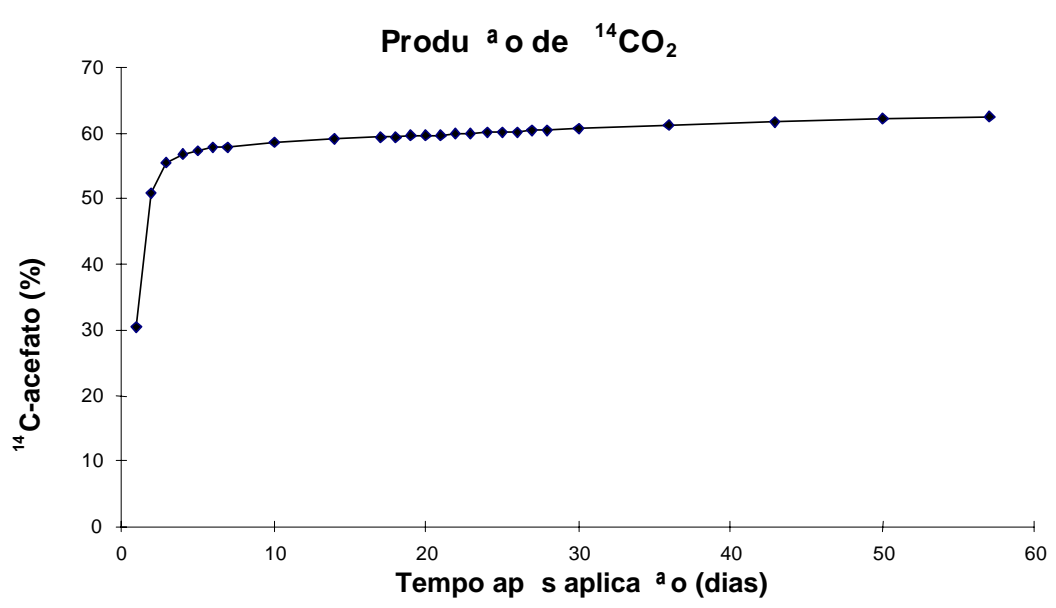

A análise do solo (coletado após os períodos de incubação pré-definidos) revelou que houve dissipação dos resíduos presentes no solo, dos resíduos extraíveis e dos resíduos ligados, sendo os últimos dissipados lentamente em função do tempo (Tabela 3).

Quanto à análise dos resíduos ligados, logo após os 3 dias da aplicação, verificou-se redução de $25 \%$ para cerca de $16 \%$ até o final do estudo (Tabela 3 ).

TABELA 3 - PORCENTAGEM DE RESíDUOS ENCONTRADOS NOS EXTRATOS E NA COMBUSTÃO DO SOLO APÓS CADA PERÍODO DE INCUBAÇÃO DO PRIMEIRO TESTE (64 DIAS)

\begin{tabular}{cccc}
\hline $\begin{array}{c}\text { Tempo ap s } \\
\text { aplica } \mathbf{~ o}\end{array}$ & $\begin{array}{c}\text { Res duos } \\
\text { extra veis }\end{array}$ & $\begin{array}{c}\text { Res duos } \\
\text { ligados }\end{array}$ & Total \\
\hline \hline (dias) & & $----\left(\% \pm\right.$ desvio-padr ${ }^{\mathrm{a}}$ o) & ------ \\
\hline 0 & $75,82 \pm 0,73$ & $28,13 \pm 3,64$ & $103,99 \pm 4,31$ \\
3 & $1,93 \pm 0,16$ & $25,26 \pm 0,64$ & $27,19 \pm 0,66$ \\
7 & $0,71 \pm 0,42$ & $22,68 \pm 1,83$ & $23,55 \pm 1,69$ \\
14 & $0,56 \pm 0,30$ & $21,39 \pm 0,96$ & $22,08 \pm 1,00$ \\
28 & $0,49 \pm 0,30$ & $23,42+3,03$ & $24,02 \pm 2,99$ \\
64 & $0,37 \pm 0,27$ & $16,38 \pm 1,43$ & $16,74 \pm 1,55$ \\
\hline
\end{tabular}

O segundo teste realizado confirmou a rápida mineralização do ${ }^{14} \mathrm{C}$-acefato (Figura 5), tendo sido dissipado cerca de $72,1 \%$ nas 100 primeiras horas.

Analisando mais detalhadamente o gráfico, observa-se que o processo apresentou duas cinéticas distintas. No cálculo da meia-vida $\left(T_{1 / 2}\right)$ pela porcentagem do ${ }^{14} \mathrm{C}$-inseticida remanescente no solo, ao longo do experimento (Figura 6), foram consideradas as duas inclinações da curva. Para a primeira cinética do processo obteve-se a meia-vida de 18,9 dias, enquanto que para a segunda cinética 1.386 dias. Segundo a tabela de classificação de persistência de agrotóxicos (BRASIL, 1997), a primeira cinética indica agrotóxico não-persistente e a segunda agrotóxico altamente persistente. WEBER (1994) fixou em 2 dias a meia-vida do acefato, portanto, persistência muito baixa ou não-persistente (<10 dias). Já MONTGOMERY (1993) considera a meia-vida do acefato dependente da acidez do meio 
(em água), ou seja, à medida que o meio se torna mais ácido ocorre aumento no tempo de persistência do agrotóxico $\left(\mathrm{T}_{1 / 2}=2,5\right.$ dias para $\mathrm{pH} 9 \mathrm{e} \mathrm{T}_{1 / 2}=29,6$ dias para $\left.\mathrm{pH} 3\right)$. THOMSON (1994-5) e WORTHING e HANCE (1991) apresentam o acefato como inseticida de persistência moderada, com atividade residual de 6-9 dias e 10-15 dias, respectivamente. É provável que não tenham lembrado do fato do inseticida acefato transformar-se em metamidofós e outros metabólitos. Isso pode ocorrer não só no solo, mas em qualquer meio como plantas e insetos (BULL, 1979; FRANK et al., 1984). Os metabólitos podem apresentar persistência maior que o seu composto parental. Por exemplo, o metamidofós em meio aquoso tem meia-vida de 309 dias em pH 5,0 e de 3 dias em pH 9,0 (USEPA, 1998). Apesar disso, alguns autores (BOUCHARD e LAVY, 1982; HADJIDEMETRIOU, IWATA e GONTHER, 1985) encontraram cinética de primeira ordem do acefato sem a formação de metamidofós em seus estudos com plantas. Em contrapartida, FRANK et al. (1984) em estudo de resíduos de acefato em feijão, cenoura, aipo, alface, pimentão, batata, morango e tomate, e ANTONIOUS e SNYDER (1994) detectaram formação de metamidofós como produto metabólico.

FIGURA 5 - MINERALIZAÇÃO DO ${ }^{14} \mathrm{C}$-ACEFATO EM 14 DIAS

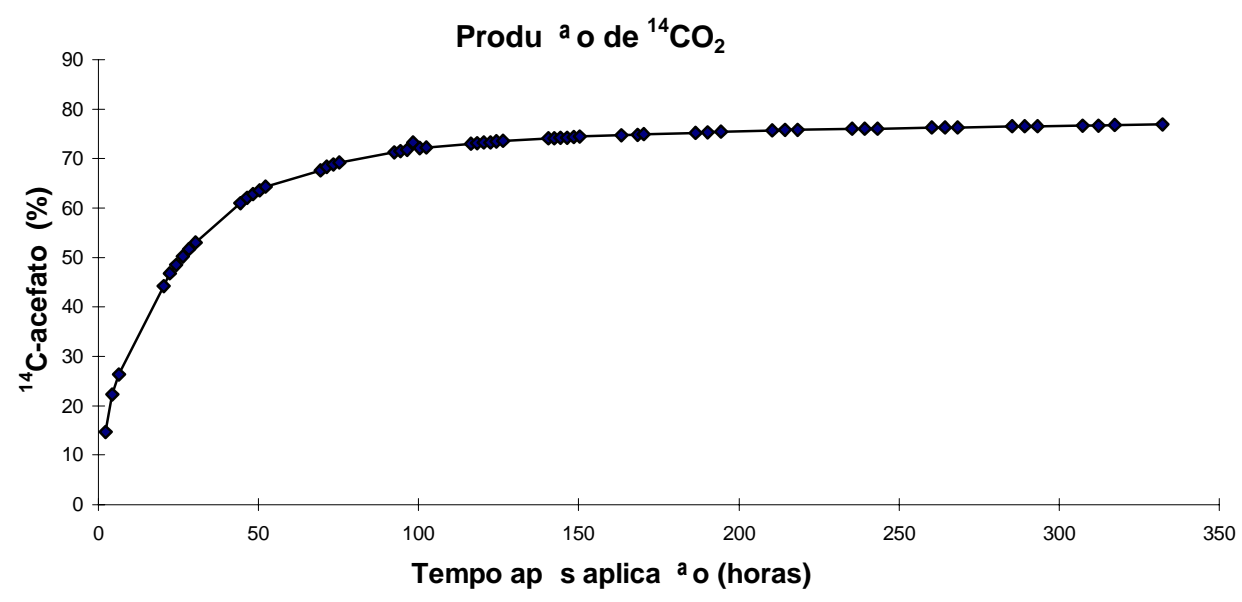

\section{FIGURA $6-{ }^{14} \mathrm{C}$-RESÍDUOS REMANESCENTES NO SOLO APÓS ADIÇÃO DE ${ }^{14} \mathrm{C}$-ACEFATO AO SOLO DOS FRASCOS BIOMÉTRICOS NO TESTE DE MINERALIZAÇÃO DE 14 DIAS - EQUAÇÃO BI-EXPONENCIAL COM R² $=0,9979$}

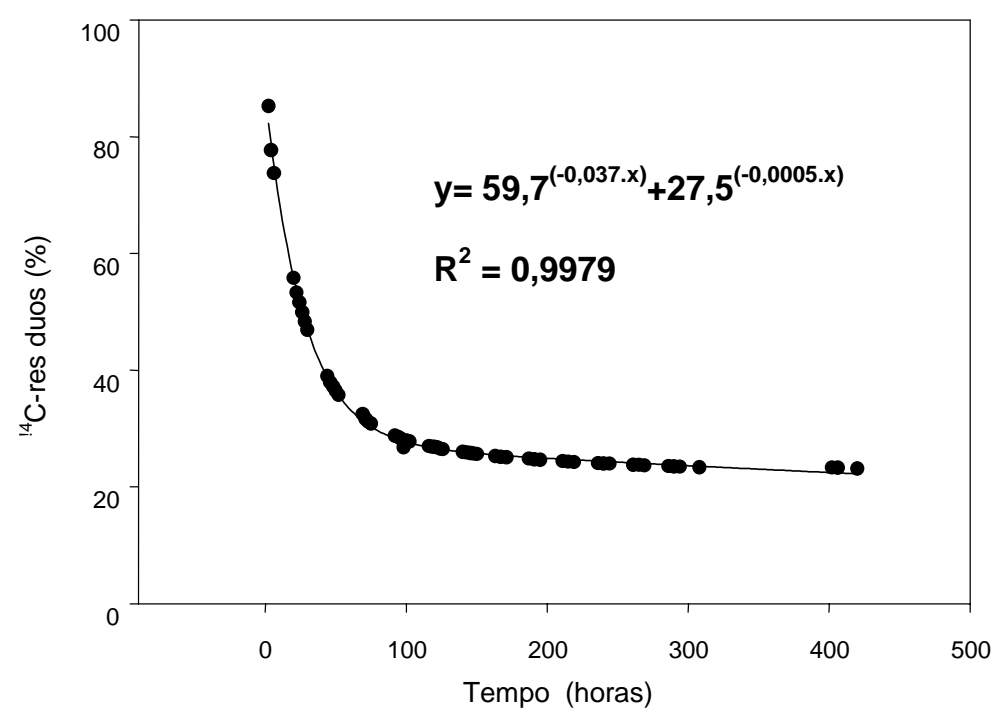


Análises do solo, após os 14 dias de incubação nos frascos biométricos, indicaram que dos $30,03 \pm 1,12 \%$ de radiocarbono remascente no solo, $29,14 \pm 0,69 \%$ permaneciam na forma de resíduo ligado e apenas $0,88 \pm 0,14 \%$ como resíduo extraível. Tais valores ficaram muito próximos dos encontrados aos 14 dias do primeiro teste de mineralização, que durou 64 dias (Tabela 3).

As características do agrotóxico como sua estrutura molecular, alta solubilidade e baixa toxicidade, além da baixa retenção no solo podem ter facilitado sua degradação inicial e a formação de compostos fortemente retidos no solo com persistência muito longa.

Embora se discuta a possibilidade de contaminação de águas subterrâneas pelo acefato em solos arenosos, devido à sua alta persistência, a contaminação depende da lixiviação do agrotóxico. Assim, apesar de sua alta solubilidade em água e baixa retenção pelos colóides do solo, a possibilidade de contaminação de águas subterrâneas pela lixiviação do acefato no perfil do solo torna-se pouco provável pela formação de compostos altamente retidos aos colóides do solo.

\title{
4 CONCLUSÃO
}

O acefato foi pouco adsorvido ao solo estudado, contudo permaneceram resíduos ligados após as sucessivas dessorções com lenta dissipação dos mesmos.

O acefato na forma de resíduo extraível foi degradado rapidamente a $\mathrm{CO}_{2}$, entretanto os resíduos ligados não foram dissipados de forma completa até 64 dias após a aplicação.

Mesmo apresentando baixa adsorção e potencial de lixiviação em solos arenosos, o acefato não representa risco de contaminação de águas subterrâneas devido a sua curta meia-vida inicial para o solo analisado.

Estudos aprofundados com relação à qualificação dos minerais do solo, além da verificação da formação de metamidofós no estudo de mineralização, forneceriam mais dados para melhor se avaliar a persistência do acefato no solo.

\begin{abstract}
SORPTION/DESORPTION AND MINERALIZATION OF THE INSECTICIDE ACEPHATE IN THE SOIL

The sorption/desorption and mineralization of organophosphate insecticide acephate applied in tropical soil (claysoil) of Campinas-SP region of Brazil was studied. Radioanalitic techniques were used in analysis of soil, air and aqueous solution to verify the presence of ${ }^{14} \mathrm{C}$-acephate in treated soil. Adsorption and desorption isotherms were obtained by treatment of 1,2 and $4 \mathrm{~g}$ of soil with $10 \mathrm{~mL}$ of $0.01 \mathrm{~mol} . \mathrm{L}^{-1} \mathrm{CaCl}_{2}$ aqueous solution with 7 different concentrations of acephate. After $24 \mathrm{~h}$ agitation in order to reach equilibrium of soil/solution, the adsorption was determined in the extracts obtained by means of ${ }^{14}$ acephate activity by scintillation counting in the liquid. The parameters, adsorption/desorption constant $(\boldsymbol{K})$ and the exponential constant $(\boldsymbol{n})$ from Freundlich equation were obtained by linear regression, besides the distribution constant $\left(\mathbf{K}_{\mathrm{d}}\right)$ and of the adsorption coefficient normalized for organic carbon content $\left(\mathbf{K}_{\mathrm{oc}}\right)$. The mineralization study of acephate in soil was conducted in biometric flasks of Bartha, with $50 \mathrm{~g}$ of soil and $10 \mathrm{~mL} \mathrm{of} \mathrm{KOH} 0.2 \mathrm{~mol}^{-L^{-1}}$ as capture solution of ${ }^{14} \mathrm{CO}_{2}$. The degradation kinetics was followed by two experiments of 14 and 64 days. Trapping solutions aliquots and sub samples of soil, after combustion, were analyzed by scintillation counting in the liquid. The results of adsorption isotherms obtained for acephate revealed that the compound is little retained in claysoil (the constants $\mathbf{K}_{t}$ and $\mathbf{K}_{d}$ showed values less than 5 ; the $\mathbf{K}_{\text {of }}$ was of 56 ). The desorption coefficient values indicated a slow and not complete process during the experiment period. The mineralization result showed that acephate is easily degraded in the $10^{\text {th }}$ first days, with approximately $70 \%$ mineralization of the insecticide to ${ }^{14} \mathrm{CO}_{2}$. Its half life in soil presented values of 18.9 and 1.386 days.
\end{abstract}

KEY-WORDS: SOIL-DEGRADATION; INSECTICIDES; RADIOMETRIC TECHNIQUES.

\section{REFERÊNCIAS}

ANTONIOUS, G.F.; SNYDER, J.C. Residues and half-lives of acephate, methamidophos, and pirimiphos-methyl in leaves and fruit of greenhouse-grown tomatoes. Bulletin of Environmental Contaminants and Toxicology, v.52, p.141-148, 1994. 

cosolvents on adsorption of organophosphorus pesticides by soils. Chemosphere, v.27, n.8, p.1409-1417, 1993.

3 BARTHA, R.; PRAMER, D. Features of a flask and method for measuring the persistence and biological effects of pesticides in soil. Soil Science, v.100, n.1, p.68-70, 1965.

4 BOUCHARD, D.C.; LAVY, T.L. Fate of acephate in the cotton plant. Journal of Economic Entomology, v.75, p.921-923, 1982.

5 BRASIL. Ministério da Habitação, Urbanismo e Meio Ambiente. Secretaria Especial do Meio Ambiente. Secretaria de Tecnologia e Controle Ambiental. Coordenadoria de Toxicologia Ambiental. Manual de testes para avaliação da ecotoxicidade de agentes químicos. ed.rev. Brasília: SEMA-STC, 1993. Cap.E 3, p.1-14 (Teste para avaliação da adsorção/ dessorção).

6 BRASIL. Ministério da Habitação, Urbanismo e Meio Ambiente. Secretaria Especial do Meio Ambiente. Secretaria de Tecnologia e Controle Ambiental. Coordenadoria de Toxicologia Ambiental. Manual de testes para avaliação da ecotoxicidade de agentes químicos. ed.rev. Brasília: SEMA, STC, 1997. cap.E 1.2, p.1-18. (Biodegradabilidade em solos)

7 BULL, D.L. Fate and efficacy of acephate after application to plant and insects. Journal of Agriculture and Food Chemistry, v.27, n.2, p.268-272, 1979.

8 BURCHILL, S.; HAYES, M.H.B.; GREENLAND, D.J. Adsorption of Organic Molecules. IN: GREENLAND, D.J.; HAYES, M.H.B. The chemistry of soil processes. Chichester: John Wiley, 1981. cap. 6, p. 221-400.

9 FRANK, R.; RITCEY, G.; BRAUN, H.E.; McEWEN, F.L. Disappearance of acephate residues from beans, carrots, celery, lettuce, peppers, potatoes, strawberries, and tomatoes. Journal of Economic Entomology, v.77, p.11101115, out. 1984

10 HADJIDEMETRIOU, D.G.; IWATA, Y.; GUNTHER, F.A. Analysis and dissipation of dislodgable residues of acephate, dimethoate and formetanate hydrochloride on citrus foliage. Pesticide Science, v.16, p.302-310, 1985.

11 HANCE, R.J. Adsorption and bioavailability. IN: GROVER, R. (Ed.) Environmental chemistry of herbicides. Boca Raton: CRC Press, 1988. cap.1, p.1-19.

12 HAQUE, R. Role of adsorption in studying the dynamics of pesticides in a soil environment. IN: HAQUE, R.; FREED, V.H. Environmental dynamics of pesticides. New York: Plenum, 1975. p.97-114.

13 IGLESIAS-JIMÉNEZ, E.; SÁNCHEZ-MARTÍN, M.J.; SÁNCHEZ-CAMAZANO, M. Pesticide adsorption in a soil-water system in the presence of surfactants. Chemosphere, v.32, n.9, p.1771-1782, 1996.

14 KOSKINEN, W.C.; CHENG, H.H. Effects of experimental variables on 2,4,5-T adsoption-desorption in soil. Journal of Environmental Quality, v.12, n.3, p.325-330, 1983.

15 LAVORENTI, A. Comportamento dos herbicidas no meio ambiente. In: WORKSHOP BIODEGRADAÇÃO, out. 1996, Campinas. Anais... Campinas: Embrapa-Cnpma, 1986. p.81-115.

16 LUCHINI, L.C. Adsorção-dessorção dos herbicidas paraquat, diuron e 2,4-D em seis solos brasileiros. Piracicaba, 1987. 91 f. Dissertação (Mestrado) - Escola Superior de Agricultura "Luiz de Queiroz", Universidade de São Paulo.

17 LUCHINI, L.C. Adsorptive behaviour of herbicides in Brazilian soils. Arquivos do Instituto Biológico, v.64, n.1, p.43-49, 1997.

18 LUCHINI, L.C.; LORD, K.A.; RÜEGG, E.F. Sorção e dessorção de inseticidas em solos brasileiros. Ciência e Cultura, v.33, n.1, p.97-101, jan. 1981.

19 MARCHETTI, M. Dinâmica do inseticida acefato em cultura de tomate em lisímetro. 2001. Piracicaba, 2001. 182 f. Dissertação (Mestrado em Ciências) - Centro Energia Nuclear na Agricultura, Universidade de São Paulo.

20 MONTGOMERY, J.H. Agrochemicals desk reference: environmental data. Boca Raton: Lewis Publishers, 1993. $1141 p$

21 RAO,P.S.C.; JESSUP, R.E. Sorption and movement of pesticides and other toxic organic substances in soils. In: KRAL, D.M. (Ed.) Chemical mobility and reactivity in soil systems. Madison, Wisconsin: Soil Science Society of America, 1983. Cap.13, p.183-201. (SSSA Special Publication, 11).

22 RODRíGUEZ-GONZALO, E.; SÁNCHEZ-MARTIN, M.J.; SÁNCHEZ-CAMAZANO, M. Determination of acephate by liquid chromatography in the presence of aqueous soil extracts. Journal of Chromatography, v.585, p.324-328, 1991. 
SÁNCHEZ-CAMAZANO, M.; GONZALEZ-POZUELO, J.M.; SANCHEZ-MARTÍN, M.J.; CRISANTO, T. Adsoption and mobility of acephate in soils. Ecotoxicology and Environmental Safety, v.29, p.61-69, 1994.

24 SCARONI, I; CALDARI, C. Studio di punti critici per la ricerca di residui di fitofarmaci organofosforici negli alimenti. Industrie Alimentari, v.363, n.36, p.1131-1134, out. 1997.

25 SCHEUNERT, I. Transport and transformation of pesticides in soil. In: MANSOUR, M. (Ed.) Fate and prediction of environmental chemicals in soils, plants, and aquatic systems. Boca Raton: Lewis, 1993. Cap.1, p.1-22.

26 SCHWARZENBACH, R.P.; GSCHWEND, P.M.; IMBODEN, D.M. Sorption: solid-aqueous solution exchange. In: SCHWARZENBACH, R.P.; GSCHWEND, P.M.; IMBODEN, D.M. Environmental organic chemistry. New York: John Wiley, 1993a. cap.11, p.255-341.

27 SCHWARZENBACH, R.P.; GSCHWEND, P.M.; IMBODEN, D.M. Chemical transformation reactions. In: SCHWARZENBACH, R.P.; GSCHWEND, P.M.; IMBODEN, D.M. Environmental organic chemistry. New York: John Wiley, 1993b. cap.12, p.342-435.

28 SOMASUDARAN, L.; COATS, J.R. Pesticide transformation products in the environment. In: SOMASUDARAN, L.; COATS, J.R. Pesticide transformation products: fate and significance in the environment. Oxford: ACS Symposium Series, 1991. Cap. 1, p. 2-9.

29 THOMSON, W.T. Agricultural chemicals: insecticides, acaricides and ovicides. Fresno: Thomson, $1994 / 95$. p.210-211.

30 USEPA. United States Environmental Protection Agency. Pesticide environmental fate on line summary: methamidophos. In: EXTENSION toxicology network: methamidophos. 1998. Disponível em <http://extoxnet.orst.edu/ pips/methamid.htm>. Acesso em jan. 2001.

31 YEN, J.H.; LIN, K.H.; WANG, Y.S. Potential of the insecticides acephate and methamidophos to contaminate groundwater. Ecotoxicology and Environmental Safety, v.45, n.1, p.79-86, jan. 2000.

32 WEBER, J.B. Properties and behaviour of pesticides in soil. In: HONEYCUTT, R.C. (Ed.). Mechanisms of pesticide movement into ground water. Boca Raton: Lewis, 1994. Cap. 2, p.15-41.

33 WORTHING, C.R.; HANCE, R.J. The pesticide manual: a world compendium. 9. ed. Farnham: BCPC, 1991. $1141 p$.

\section{AGRADECIMENTOS}

Agradeço ao CNPq pela bolsa de mestrado, aos colegas do Laboratório de Ecologia de Agroquímicos do Instituto Biológico de São Paulo pelo apoio e ao pesquisador Claudio Spadotto (EMBRAPA - Meio Ambiente) pelo programa estatístico. 